\title{
Serum Proteome Pool Changes in Type 2 Diabetic Patients Treated with Anakinra
}

\author{
Mette Koefoed - Claus M. Larsen • Mirjam V. Faulenbach • Allan Vaag • \\ Jan A. Ehses • Marc Y. Donath • James Norton McGuire • Flemming Pociot • \\ Thomas Mandrup-Poulsen
}

Published online: 28 September 2010

(C) Springer Science+Business Media, LLC 2010

\begin{abstract}
Introduction High glucose concentrations induce the production of IL-1 $\beta$ in human pancreatic beta cells leading to impaired insulin secretion, decreased cell proliferation and apoptosis. Blockade of IL-1 signalling with the recombinant human IL-1 receptor antagonist anakinra reduces $\mathrm{HbA}_{1 \mathrm{c}}$ in patients with type 2 diabetes.
\end{abstract}

Electronic supplementary material The online version of this article (doi:10.1007/s12014-010-9056-x) contains supplementary material, which is available to authorized users.

M. Koefoed $\cdot$ C. M. Larsen · A. Vaag · J. N. McGuire · F. Pociot • T. Mandrup-Poulsen

Steno Diabetes Center and Hagedorn Research Institute,

Gentofte, Denmark

M. V. Faulenbach · J. A. Ehses $\cdot$ M. Y. Donath

University Hospital Zurich and Center for Integrative Human

Physiology,

Zurich, Switzerland

F. Pociot

CRC MAS, University of Lund,

Malmö, Sweden

T. Mandrup-Poulsen

Institute for Biomedical Sciences, University of Copenhagen,

Copenhagen, Denmark

T. Mandrup-Poulsen

Department of Medicine and Surgery, Karolinska Institute,

Stockholm, Sweden

T. Mandrup-Poulsen $(\square)$

Department of Translational Diabetology,

Hagedorn Research Institute,

1 Niels Steensensvej,

DK 2820 Gentofte, Denmark

e-mail: tmpo@hagedorn.dk
The aims of the present study were to identify: (1) candidate surrogates for improved glycemia in type 2 diabetic patients following treatment with anakinra, (2) proteins that change serum concentration because of anakinra treatment and (3) candidate biomarkers that may predict improved glycemia in type 2 diabetic subjects treated with anakinra.

Methods Surface-enhanced laser desorption/ionisation time-of-flight mass spectrometry was used to analyse serum from 67 type 2 diabetic patients who had received either placebo or anakinra for 13 weeks. Immunodepletion with magnetic protein $G$ bead-coupled antibodies were used to identify three proteins and Western blotting confirmed the biomarker concentration pattern of four proteins.

Results Twelve proteins, including transthyretin (TTR) and transferrin (Tf), were identified as candidate surrogates for improved glycemia. Six proteins, including retinol-binding protein 4 (RPB4) and a protein tentatively identified as modified apolipoprotein-A1 (apo-AI), increased expression as a consequence of anakinra treatment and four proteins were candidate biomarkers that may predict improved glycemia following anakinra treatment. Furthermore, we found increased RBP4 to be associated with improved beta cell secretory function and increased TTR, RBP4 and modified apo-AI (peak at 28,601 Da) to be associated with decreased inflammation.

Conclusions Anakinra-induced changes in the serum proteome pool associated with a decreased cardiovascular disease risk, reduced inflammation and improved beta cell secretory function.

Keywords Biomarkers · Proteomics · Inflammation · SELDI-TOF-MS · Transthyretin $\cdot$ Retinol binding protein $4 \cdot$ Transferrin $\cdot$ Apolipoprotein-AI 


$\begin{array}{ll}\begin{array}{ll}\text { Abbreviations } \\ \text { apo-AI }\end{array} & \text { Apolipoprotein-AI } \\ \text { BG } & \text { Blood glucose } \\ \text { BMI } & \text { Body mass index } \\ \text { CRP } & \text { C-reactive protein } \\ \text { CVD } & \text { Cardiovascular disease } \\ \text { IL-1Ra } & \text { IL-1-receptor antagonist } \\ \mathrm{m} / z & \text { Mass/charge } \\ \text { RBP4 } & \text { Retinol binding protein } 4 \\ \text { ROC } & \text { Receiver operator characteristic } \\ \text { SELDI-TOF-MS } & \text { Surface-enhanced laser desorption/ } \\ & \text { ionisation time-of-flight mass } \\ & \text { spectrometry } \\ \text { SPA } & \text { Sinapinic acid } \\ \text { Tf } & \text { Transferrin } \\ \text { TG } & \text { Triglyceride } \\ \text { TTR } & \text { Transthyretin } \\ \text { FFA } & \text { Free fatty acids }\end{array}$

\section{Introduction}

IL-1 $\beta$ is a proinflammatory cytokine that has been implicated as an effector molecule of inflammatory beta cell destruction leading to type 1 diabetes [1]. Intra-islet production of IL-1 $\beta$ may also play a role in the pathogenesis of type 2 diabetes [2]. In pancreatic sections obtained from type 2 diabetic patients, beta cells express IL-1 $\beta$ [3]. High glucose levels increase beta cell production and release of IL-1 $\beta$ via reactive oxygen-dependent activation of the inflammasome [4], leading to impaired insulin secretion, decreased cell proliferation and apoptosis in human islets in vitro [3].

IL-1-receptor antagonist (IL-1Ra) is a naturally occurring competitive inhibitor of IL-1 binding to the IL-1 receptor. IL$1 \mathrm{Ra}$ protects human beta cells from glucose-induced functional impairment and apoptosis [3]. The expression of IL-1Ra is reduced in pancreatic islets in patients with type 2 diabetes [5]. Blockade of IL-1R with the recombinant human IL-1Ra anakinra reduced $\mathrm{HbA}_{1 \mathrm{c}}$, enhanced Cpeptide secretion, reduced the proinsulin to insulin ratio and reduced the levels of IL- 6 and C-reactive protein (CRP) in type 2 diabetic patients [2]. Insulin resistance, insulin-regulated gene expression in skeletal muscle, serum adipokine levels and body mass index (BMI) did not change during treatment with anakinra $[2,6]$.

Genetically determined low baseline IL-1Ra, body weight and BMI have been identified as predictors of improved glycemia following anakinra treatment [2, 7]. Body weight and BMI were interpreted as surrogates for drug distribution volume, suggesting that higher systemic drug concentrations predict a glycemic response. Neither baseline nor changes in levels of CRP or IL-6 were correlated with changes in $\mathrm{HbA}_{1 \mathrm{c}}$. Because not every patient responded to the same extent to the treatment, it is important to investigate if there are other predictors for the glycemic response of the individual type 2 diabetic patients to IL-1 antagonism. Our aims were therefore: (1) to find candidate surrogates for improved glycemia in type 2 diabetic patients treated with anakinra, (2) to find proteins that change serum concentration as a consequence of anakinra treatment and (3) to find more precise or useful biomarkers that may predict improved glycemia in type 2 diabetic patients treated with anakinra.

For this purpose, we analysed sera from the described study [2] by surface-enhanced laser desorption/ionisation time-of-flight mass spectrometry (SELDI-TOF-MS) which separates proteins and peptides according to their mass-tocharge ratio. SELDI-TOF-MS provides a sensitive and reproducible method to detect and resolve multiple proteins bound to protein chip arrays. The platform has been used in several large-scale proteomic clinical studies on plasma, serum, cerebrospinal fluid and urine [8-11]. It has further been used as a tool in the development of diagnostic assays of various diseases, including several types of cancer, heart infarction, Alzheimer's disease, rheumatoid arthritis and chronic alcoholism [9, 12-18].

\section{Methods}

Patients Patient samples originate from a placebocontrolled, double-blind, parallel-group study involving 70 individuals with type 2 diabetes at two centres (31 patients in Denmark and 39 patients in Switzerland) [2]. Patients received either once-daily recombinant human IL-1Ra (100 mg of anakinra) or placebo by subcutaneous selfadministration for 13 weeks. Baseline and 13th week sera from all 67 patients who completed the primary study were analysed in this study. Sera were stored at $-80^{\circ} \mathrm{C}$ until and had been thawed three times prior to analysis.

Study Design The patients were divided as follows: (1) anakinra responders $(n=21),(2)$ anakinra non-responders $(n=13)$, (3) placebo "responders" $(n=10)$ and (4) placebo "non-responders" $(n=23)$. Response was defined as any reduction in $\mathrm{HbA}_{1 \mathrm{c}}$ following 13 weeks of intervention. The number of responders was 21 of 34 patients in the anakinra group and 11 of 33 in the placebo group [2]. Glycosylated haemoglobin A1c $(\mathrm{HbAlc})$ is the fraction of the normal and abundant haemoglobin A variant which is irreversibly and non-enzymatically glycosylated by exposure to glucose in plasma. The rationale for using $\mathrm{HbAlc}$ as primary endpoint in the anakinra in type 2 diabetes trial was that $\mathrm{HbA1c}$ correlates well with average plasma glucose and thus reflects the ambient mean plasma glucose concentration 
over the past 4-12 weeks with a major contribution from the first 4-week period [19]. This endpoint was therefore well suited to investigate the effect of anakinra on metabolic control after 4 and 13 weeks of therapy, whereas acute $(<1$ week) effects on metabolic control were studied by the means of home monitoring of fasting blood glucose.

To identify candidate surrogate biomarkers for improved glycemia, we compared the spectra at baseline with spectra at 13 weeks of treatment in group 1 . To control for differences that were unrelated to the primary clinical outcome, the same comparison was done in group 2. Only peaks that were significant in group 1 were carried forward. To control for the placebo effect, the same comparison was done in the placebo groups. To identify changes in the proteome pool from baseline to 13th week as a consequence of anakinra treatment, we sought spectrum differences between baseline and 13 weeks of treatment in all anakinra-treated patients (groups 1 and 2). To exclude the placebo effect, we ensured that the same differences were not found in the placebo groups (groups 3 and 4). The protein changes due only to anakinra treatment were those significant only in the anakinra group. Protein changes also significant as surrogates for outcome were deleted. To identify biomarkers that may predict improved glycemia in type 2 diabetic patients treated with anakinra, we looked for spectrum differences between groups 1 and 2 at baseline. To eliminate the placebo effect, we ensured that the same differences were not found between groups 3 and 4 . Biomarkers that may predict improved glycemia to the treatment were those unique for group 1.

Albumin Depletion and SELDI-TOF-MS Analysis All patient serum samples were depleted in a high throughput automated system. The eluate from the depletion step for each patient was analysed using five different array conditions: CM10 pH 7, Q10 pH 5, Q10 pH 9, H50 and IMAC30-Nickel. Arrays were prepared using an automated system and read in a PCS-4000 instrument (Bio-Rad) with two laser settings. A high laser setting focused on larger proteins (4,000-100,000 Da) while a low setting was employed for peptides $(2,000-10,000 \mathrm{Da})$. For each patient, a single biological replicate was albumin depleted and analysed in duplicate by SELDI-TOF-MS. Mass values in this study are assumed to have a single charge and therefore are reported as Da. Detailed protocols for albumin depletion, array preparation and array reading are included as supplementary information.

Identification of Proteins with Protein G Beads Albumindepleted human reference serum was produced using blue trisacryl resin and diluted in Tris-Buffered Saline Tween-20 (TBST). Protein $\mathrm{G}$ beads (Invitrogen) were added to different Eppendorf tubes and washed in phosphate- buffered saline (PBS). Anti-transthyretin (TTR), antiretinol binding protein 4 (RPB4) and anti-transferrin (Tf) antibodies (DakoCytomation, Glostrup, Denmark) were diluted in PBS, added to the tubes and shaken for $30 \mathrm{~min}$ at room temperature. The beads were washed three times in TBST and incubated with diluted albumin-depleted serum for $30 \mathrm{~min}$ with shaking. The unbound sample was analysed on SELDI arrays using the specified protocol. A control was made using the same protocol but without antibody.

Quantitative Western Blotting Sera from the patients with the highest, an intermediate and the lowest peak amplitude of TTR, RBP4, Tf and 28,601 Da protein (to which we assigned the preliminary identity of modified apolipoprotein$\mathrm{AI}$ (apo-AI)) were diluted in TBST (TTR, apo-AI and Tf) or water (RBP4) and heated for $5 \mathrm{~min}$ at $80^{\circ} \mathrm{C}$. Molecular weight markers (Bench Marker and Magic Marker (Invitrogen)) and diluted sera mixed with loading buffer were electrophoresed on $10 \%$ Bis-Tris SDS-PAGE gels (Invitrogen) and transferred to nitrocellulose membranes (Invitrogen). Western blotting was performed with non-fat milk (Merck) as a blocking agent. Blots were incubated overnight at $4^{\circ} \mathrm{C}$ with primary antibody (anti-TTR, anti-RBP4, anti-apo-AI or anti-Tf) and for $1 \mathrm{~h}$ at room temperature with polyclonal anti-Rabbit IgG (Cell Signalling, Danvers, MA, USA). The membranes were placed for approximately $5 \mathrm{~min}$ in developing agent (LumiGlo reagent, Cell Signaling, Danvers, MA). Chemiluminescence was used as the output signal. Imaging of the membranes was performed using a LAS-3000 Image Reader (Fuji Film, Tokyo, Japan).

Statistical Analyses The software R version 2.7.0 (see www.r-project.org) was used for the statistical analysis. A Wilcoxon signed-rank test (significance level of $p<0.05$ ) was used to test for differences in protein levels to identify biomarkers as surrogates for improved glycemia, to identify changes in the proteome pool from baseline to 13 week as a consequence of anakinra treatment and to test for significant changes in clinical markers. To identify biomarkers that may predict improved glycemia in patients treated with anakinra, both a Mann-Whitney test and an estimation of the area under the receiver operator characteristic curve (AUC) with significance levels of $p<0.05$ and AUC> 0.69 , respectively, were employed.

CiphergenExpress Data Manager was used to find and label protein peaks. To confirm that all peaks were labelled correctly, the peaks representing candidate biomarkers were manually relabelled and the statistical tests (Wilcoxon and Mann-Whitney) were repeated. Some of the peaks were represented in more than one SELDI array condition; only one of them was saved for further analysis. If the peak was represented in both the "high mass" and the "low mass" 
reading protocols, the peak in the "high mass" reading protocol was retained. If the peak was represented in different SELDI array conditions, the peak in the condition with best $\mathrm{CV}$ value was retained.

A correlation test was performed on all the remaining peaks where $r>0.7$ was considered significant as a reasonable compromise between stringency and sensitivity. Peaks that were strongly correlated and had Da values that were either twice or half the value of each other were considered to be either a dimer or a double charge of the protein. Only the monomer, the whole protein, was kept for further analysis. Other peaks that were strongly correlated were considered to be different modifications of the same protein. A correlation test where $p<0.05$ vas considered significant was also used to test for correlation between changes in serum protein concentration and changes in clinical markers.

Resampling of the results was performed in $\mathrm{R}$ using a permutation test with a significance level of $p<0.05$ was performed on the remaining peaks. Only peaks that were considered significant after resampling were kept for further analysis.

\section{Results}

SELDI Analysis Analysis of the 134 samples run in duplicate produced 539 peak clusters. When the protein peak intensities were compared between the groups, 148 of them showed significantly different serum levels in at least one of the three tests: 49 potentially useful as surrogates for improved glycemia, 76 that changed expression because of treatment with anakinra and 23 that may predict glycemic response to the treatment. After peak alignment, resampling and deletion of peaks present in more than one condition the following numbers of candidate biomarkers were found: 12 potentially useful as surrogates for improved glycemia (Table 1), six that changed expression because of treatment with anakinra (Table 2) and four that may predict glycemic response to the treatment (Table 3 and 4).

A correlation test showed the following protein peaks to be highly correlated to one another: 7,048, 14,089, 14,300, 14,507 and $27,788 \mathrm{Da}(r=0.7-0.8) ; 13,944,14,300$ and $27,788 \mathrm{Da}(r=0.7-0.8) ; 39,866$ and $79,628 \quad(r=0.83$; Table 1) and 21,086 and 21,278 Da ( $r=0.72$; Table 2), respectively. A strong correlation is an indication that the peaks are biochemically related and could represent modifications with the same expression pattern or species created in the ionisation process (e.g., dimers or multiply charged peaks) that appear at different mass readings but represent the same protein [20].
Identification of Proteins with Protein G Beads In this type of proteomics study emphasis is placed on analysing whole proteins from individual patients. The protein peaks that were investigated were chosen based on the fact that they were judged to be high quality peaks in our data set. Their identities were not known ahead of time but the peaks at $13,752,21,086,28,601$ and 79,628 had masses very close to those of TTR, RBP4, apo-AI and Tf, respectively. We therefore assigned these preliminary identities to the proteins. Depletion with magnetic protein $G$ beads and specific antibodies confirmed the identities of TTR, RBP4 and Tf (Fig. 1). The antibodies against apo-AI did not deplete the large peak at 28,100 $\mathrm{Da}$ or the shoulder at $28,601 \mathrm{Da}$ that are visible in the upper spectrum of Fig. 1c, which depicts depletion of the peak at $27,788 \mathrm{Da}$ by the anti-TTR antibody.

Western Blotting Western blotting verified the concentration patterns of TTR, RPB4, ApoA1 and Tf found in the SELDITOF-MS analysis. For each biomarker, we analysed three different patient samples: a serum with the lowest peak amplitude, one with intermediate peak amplitude and one with the highest peak amplitude of the respective proteins. The patient with the lowest peak amplitude had the lowest concentration quantified by Western blotting and the patient with the highest peak amplitude had the highest concentration (Fig. 2).

Correlation with Clinical Parameters In group 1 and 2, changes in RBP4 were negatively correlated to IL-6, CRP and stimulated insulin levels and positively correlated to plasma glucose after $120 \mathrm{~min}$ during OGTT and fasting insulin levels. Changes in the $28,601 \mathrm{Da}$ protein were negatively correlated to IL-6 and CRP. The 7,042 Da protein was positively correlated to free fatty acids (FFA) and negatively correlated to CRP, the $8,208 \mathrm{Da}$ protein was positively correlated to TG and cholesterol and the 20,990 Da protein was negatively correlated to IL-6 and CRP. In group 1 , changes in TTR were negatively correlated with IL- 6 and CRP, changes in the 2,740 Da protein were positively correlated to fasting insulin and negatively correlated to IL-6, changes in the $39,866 \mathrm{Da}$ protein were positively correlated to stimulated C-peptide and the 56,878 Da protein was negatively correlated to CRP, IL-6 and positive correlated to fasting insulin levels. In group 1 baseline. the 5,898 $\mathrm{Da}$ protein was positively correlated to high-density lipoprotein (HDL) and CRP and negatively correlated to FFA, the 8,741 Da protein was positively correlated to TG and FFA and the 50,123 Da protein was negatively correlated to LDL, TG, cholesterol and $B G$. None of the changes in peak amplitude were significantly correlated to $\mathrm{HbA}_{1 \mathrm{c}}$ or fasting C-peptide (Table 4). 
Table 1 Biomarkers potentially useful as surrogates for clinical outcome

\begin{tabular}{|c|c|c|c|c|}
\hline Peak (Da) & Protein identity & Biomarker change at 13 weeks & $\begin{array}{l}p \text { value for responder }{ }^{\mathrm{a}} \\
(n=21, \text { Wilcoxon })\end{array}$ & $\begin{array}{l}p \text { value for responder }{ }^{\mathrm{a}} \\
(n=21, \text { permutation test })\end{array}$ \\
\hline 2,740 & Unknown & Up & 0.016 & 0.009 \\
\hline 7,048 & Unknown & Up & 0.022 & 0.011 \\
\hline 13,752 & Transthyretin & Up & 0.002 & 0.001 \\
\hline 13,944 & Transthyretin (modified) & Up & 0.006 & 0.004 \\
\hline 14,089 & Transthyretin (modified) & Up & 0.004 & 0.003 \\
\hline 14,300 & Transthyretin (modified) & Up & 0.001 & 0.001 \\
\hline 14,507 & Transthyretin (modified) & Up & 0.001 & 0.001 \\
\hline 14,758 & Transthyretin (modified) & Up & $<0.001$ & 0.001 \\
\hline 27,788 & Transthyretin (dimer) & Up & 0.013 & 0.007 \\
\hline 39,866 & Unknown & Up & 0.042 & 0.021 \\
\hline 56,878 & Unknown & Up & 0.004 & 0.003 \\
\hline 79,628 & Transferrin & Up & 0.042 & 0.021 \\
\hline
\end{tabular}

${ }^{\mathrm{a}}$ Response was defined as any reduction in $\mathrm{HbA}_{1 \mathrm{c}}$ during the 13 week of intervention

\section{Discussion}

If all samples are treated identically, SELDI-TOF-MS is reproducible and quantitative. The method has been used successfully in large-scale proteomic studies of biomaterial from individual patients $[8-10,14,17]$. The reproducibility of SELDI-TOF-MS analysis, however, is sensitive to sample preparation and analytical conditions, which can have a significant impact on protein peak detection [20, 21]. Sample collection protocols can be flexible with regard to clotting times, transport and storage time on ice and up to three freeze/thaw cycles do not change the protein profile significantly as long as the samples are thawed on ice $[20,21]$. The critical issue is that all individual samples are treated in exactly the same way [20]. We carefully treated all the samples including controls (human reference serum) in a highly standardised way. All samples had been through three freeze/thaw cycles before SELDI analysis, were stored at $-80^{\circ} \mathrm{C}$ and thawed on ice. We obtained reproducibility with an average $\mathrm{CV}$ of $13 \%$ for Q10 pH5, which was the surface condition with the highest number of significant peaks.

One of the limitations in using SELDI-TOF-MS is the time-consuming and complicated process of identification of the significant peaks. SELDI-TOF-MS cannot sequence peptides and, therefore, is a poor platform for protein identification. To identify proteins, they must be purified, checked by SELDI using the same array surface on which it was originally identified and sequenced on another type of mass spectrometer. A more direct route to identification is through the specificity of antibodies. This method does not require other mass spectrometers but does necessitate knowledge of the identity of common SELDI-TOF-MS peaks. The identities of TTR, RBP4 and Tf were confirmed by immunodepletion of the respective proteins with magnetic protein $\mathrm{G}$ bead-coupled antibodies. When we compared the peak intensities of TTR, RBP4, ApoA1 and Tf with the intensities of the bands on Western blots, we

Table 2 Proteins altering concentration due to anakinra treatment

\begin{tabular}{|c|c|c|c|c|}
\hline Peak (Da) & Protein identity & $\begin{array}{l}\text { Biomarker change } \\
\text { at } 13 \text { weeks }\end{array}$ & $\begin{array}{l}P \text { value for treated }{ }^{\mathrm{a}} \\
(n=34, \text { Wilcoxon })\end{array}$ & $\begin{array}{l}p \text { value for treated }{ }^{\mathrm{a}} \\
(n=34, \text { permutation test })\end{array}$ \\
\hline 7,042 & Unknown & Up & 0.050 & 0.025 \\
\hline 8,208 & Unknown & Up & 0.025 & 0.013 \\
\hline 20,990 & Unknown & Up & 0.009 & 0.005 \\
\hline 21,086 & RBP4 & Up & 0.017 & 0.009 \\
\hline 21,278 & RPB4 (modified) & Up & 0.043 & 0.021 \\
\hline 28,601 & Apo-A1 ${ }^{\mathrm{b}}$ (modified) & Up & 0.007 & 0.004 \\
\hline
\end{tabular}

${ }^{\mathrm{a}}$ The treated patients received daily subcutaneous injections with $100 \mathrm{mg}$ of anakinra for 13 weeks

${ }^{\mathrm{b}}$ The final identity of the 28,601 Da protein as being modified apo-AI has not been definitively established; the preliminary identity is based on the mass and similarity to Western blot findings 
Table 3 Candidate biomarkers of clinical response to the treatment

\begin{tabular}{|c|c|c|c|c|c|}
\hline Peak (Da) & Protein identity & $\begin{array}{l}\text { Level in responder } \\
\text { vs. non responder }\end{array}$ & $\begin{array}{l}p \text { value for treated } \\
(n=34, \text { Mann- Whitney) }\end{array}$ & $\mathrm{ROC}$ & $\begin{array}{l}p \text { value for treated } \\
(n=34, \text { permutation test })\end{array}$ \\
\hline 5,898 & Unknown & High & 0.035 & 0.718 & 0.023 \\
\hline 6,663 & Unknown & Low & 0.018 & 0.750 & 0.017 \\
\hline 8,741 & Unknown & Low & 0.055 & 0.700 & 0.044 \\
\hline 50,123 & Unknown & High & 0.065 & 0.692 & 0.035 \\
\hline
\end{tabular}

found the same patterns, but not the same fold increase between patients as we found in SELDI analysis. We therefore investigated the effect on the four different peak intensities of diluting the albumin-depleted serum two-fold. We found that there was a linear correlation between the peak intensity and the albumin-depleted serum concentration, but the fold increase in peak intensity did not correspond to the fold increase in amount of protein loaded. This supported the Western blot findings.

We found a significant increase in the serum level of TTR in the anakinra responder group and TTR is therefore a candidate biomarker as surrogate for clinical outcome. As type 2 as well as type 1 diabetic patients have been found to have a decreased level of TTR compared to healthy controls [22-24], an increase in TTR might be a marker of improved metabolic control, although there was no statistical correlation between TTR and glycemic markers. Because reduced levels of TTR have been associated with inflammation [25-27] the rise in TTR might also signal decreased inflammation and we confirm that an increase in serum TTR is negatively correlated to IL-6 and CRP. However, although anakinra markedly reduced serum CRP and IL-6, these inflammatory markers were not correlated to clinical outcome $[2,7]$.

The level of RBP4 increased when type 2 diabetic patients were treated with anakinra. Plasma RBP4 is reduced in rats with spontaneous onset of type 1 diabetes [28], rats with streptozotocin-induced diabetes [29] and humans with new-onset type 1 diabetes, but the levels
Table 4 Correlation of peak data to clinical data

\begin{tabular}{ll}
\hline$\Delta$ peak intensity (Da) & Significant correlations $(p<0.05)$ \\
\hline Significant correlations in changes from baseline to 13 weeks \\
2,740 & $\Delta$ IL-6, $\Delta$ fasting insulin \\
7,048 & $\Delta$ CRP \\
13,752 & $\Delta$ IL-6, $\Delta$ CRP \\
13,944 & $\Delta$ CRP \\
14,089 & $\Delta$ IL-6, $\Delta$ CRP, $\Delta$ fasting insulin \\
14,300 & $\Delta$ IL-6, $\Delta$ CRP \\
14,507 & $\Delta$ IL-6, $\Delta$ CRP \\
14,758 & $\Delta$ IL-6, $\Delta$ CRP \\
27,788 & $\Delta$ IL-6, $\Delta$ CRP \\
39,866 & $\Delta$ C-peptide iv OGTT, $\Delta$ C-peptide total OGTT \\
56,878 & $\Delta$ IL-6, $\Delta$ CRP, $\Delta$ fasting insulin \\
79,628 & - \\
7,042 & $\Delta$ CRP, $\Delta$ FFA \\
8,208 & $\Delta$ triglycerides, $\Delta$ cholesterol \\
20,990 & $\Delta$ IL-6, $\Delta$ CRP \\
21,086 & $\Delta$ IL-6, $\Delta$ CRP, $\Delta$ fasting insulin, $\Delta$ iv insulin, $\Delta$ 120 min plasma glucose \\
21,278 & $\Delta$ IL-6, $\Delta$ CRP, $\Delta$ fasting insulin \\
28,601 & $\Delta$ IL-6, $\Delta$ CRP \\
Significant correlations at baseline & FFA, HDL, CRP \\
5,898 & - \\
6,663 & FFA, triglycerides \\
8,741 & LDL, triglycerides, cholesterol, blood glucose \\
50,123 & \\
\hline & \\
&
\end{tabular}


Fig. 1 The top spectra in a-e are derived from albumindepleted serum depleted of TTR (a-c), RBP4 (d) and Tf (e). Peaks with the masses of $13,752,13,889,13,944,14,089$, $14,300,14,507,14,758$, and 27,788 Da disappeared when one human reference serum was depleted of TTR. Protein peaks with the masses of 21,086 and 21,278 Da disappeared when one human reference serum was depleted of RBP4. The peak with the mass of 79,628 Da disappeared when one human reference serum was depleted of transferrin. The bottom spectra are controls without antibodies

\section{A:}

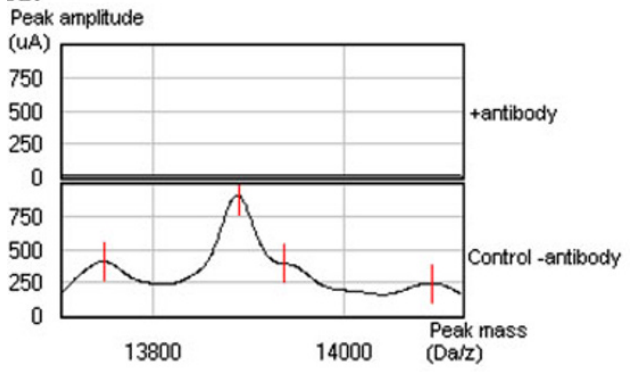

\section{C:}

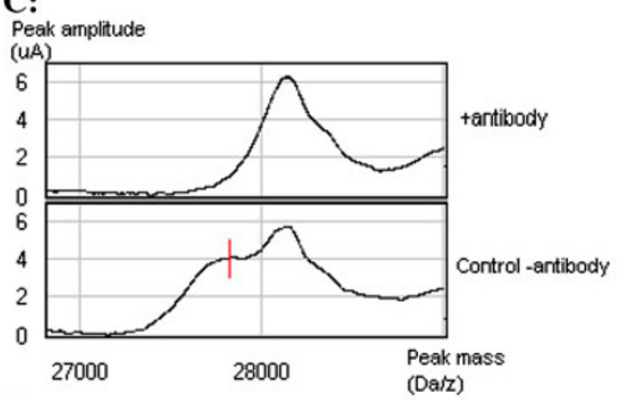

E:

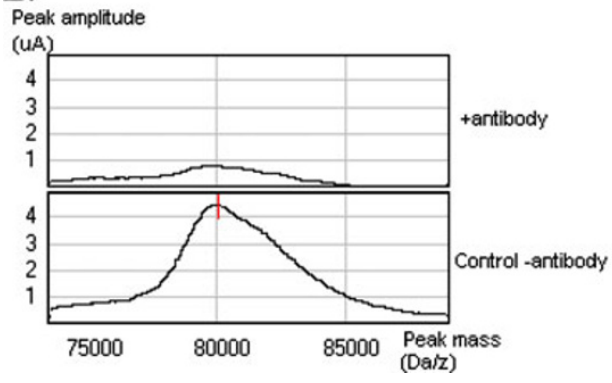

B:

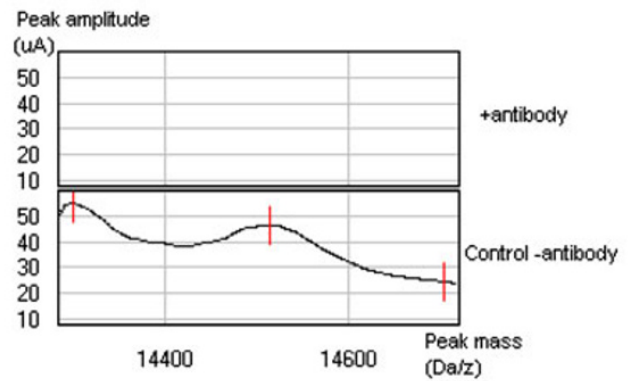

D:

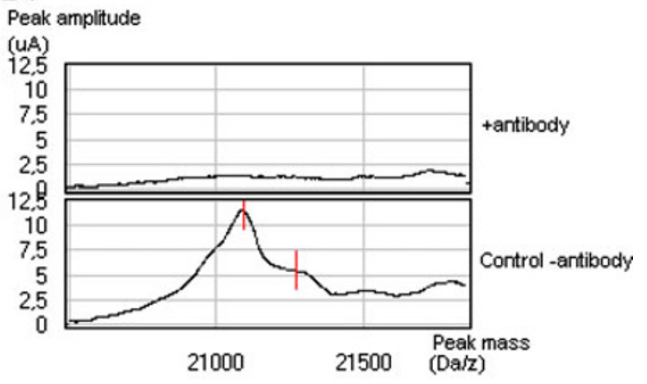

Fig. 2 Western blotting and SELDI analysis of sera from patients with low, intermediate and high peak amplitudes of apo-AI, transthyretin, RBP4, and transferrin. Western blotting verified the concentration pattern found by SELDI analysis
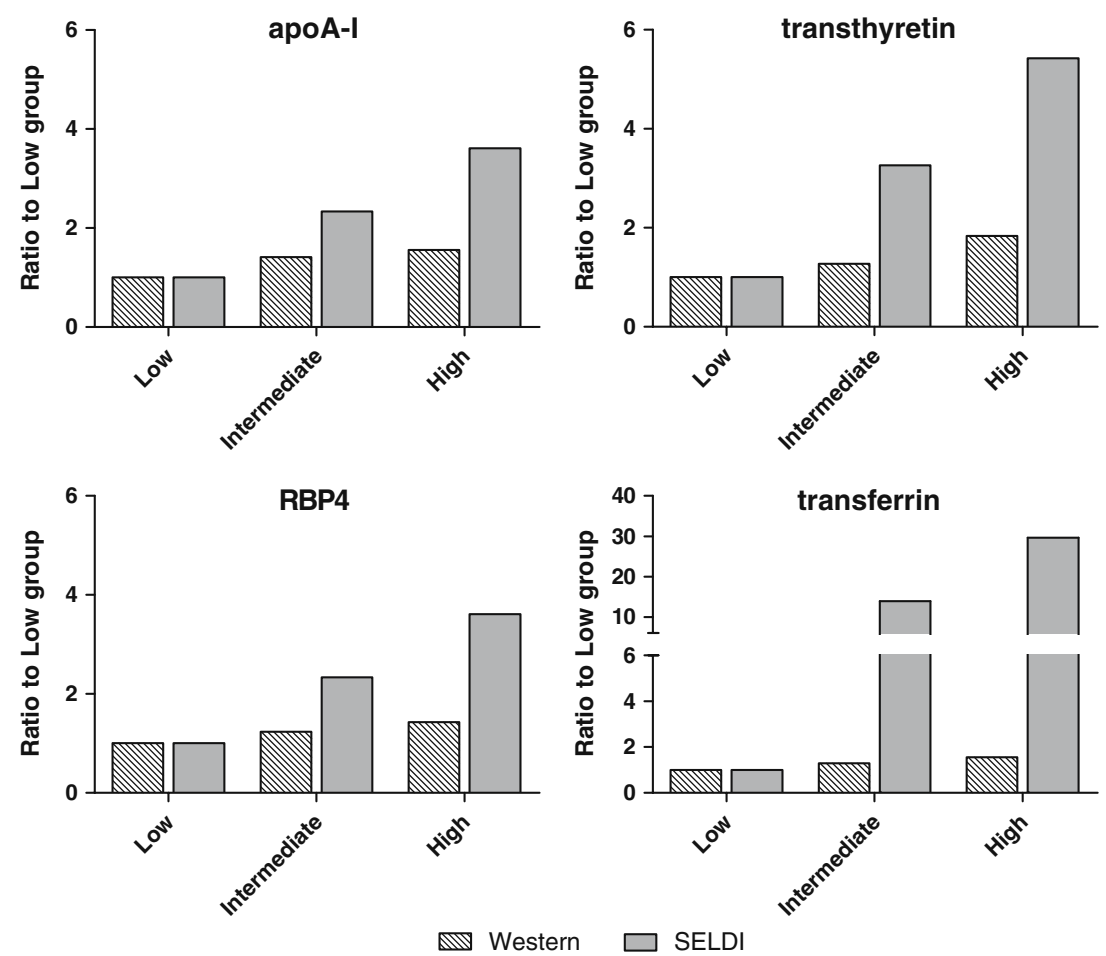
revert after insulin treatment and improved metabolic control $[22,30]$. Findings on the relationship between the level of circulating RBP4 and components of the metabolic syndrome are conflicting, with some studies reporting no correlation between RPB4 and insulin resistance [31-33] and other studies showing that RBP4 levels correlate positively with insulin resistance in type 2 diabetic subjects as well as in non-diabetic subjects [34, 35]. Elevated serum RBP4 has been positively associated with increased BMI, fasting insulin, waist-to-hip ratio, TG, systolic and diastolic blood pressure, fasting BG, $2 \mathrm{~h}$ post-OGTT glucose concentration and decreased HDL levels [34-37]. Conversely, a decreased plasma RBP4 concentration in obese female children compared with lean controls [32] and no differences in circulating RBP4 level between obese and lean postmenopausal women [31] have been observed. In a recent paper, RBP4 has been linked to insulin resistance in a non-causal manner [38]. We found increased serum RBP4 as a consequence of anakinra treatment, a positive correlation between changes in serum RBP4 and changes in fasting insulin and a negative correlation between changes in serum RBP4 and changes in CRP and IL-6.

The serum level of the 28,601 Da protein increased as a consequence of anakinra treatment. If the preliminary identity of the protein is confirmed as modified apo-AI, our results confirm a negative correlation between apo$\mathrm{AI}$ and inflammatory markers that is in accordance with apo-AI having anti-inflammatory properties [39]. If a modified form of apo-AI possesses the same antiatherogenic properties as apo-AI, it is possible that the anakinra-induced increase in the serum level of modified apo-AI may add a direct, important and unexpected benefit of anakinra treatment as a protector against cardiovascular disease (CVD).

Serum Tf increased in the anakinra responder group and is therefore a candidate surrogate biomarker for improved glycemia. Tf has been shown to be reduced in the acute phase response as well as in diabetic patients [25] with a higher risk of developing late complications. The increase observed in the anakinra responder group is postulated to be a result of decreased inflammation. The properties of $\mathrm{Tf}$ suggest that an anakinra-induced increase in serum $\mathrm{Tf}$ is associated with improvement of CVD risk because of increased iron-binding antioxidant capacity resulting in less iron-induced oxidative stress and lipid peroxidation.

In conclusion, we found 22 candidate biomarkers and identified three of them as TTR, RBP4 and Tf. Based on the mass and Western blot findings, we assigned the preliminary identification of modified apo-AI to a 28,601 Da protein. Twelve proteins (including increased serum TTR and $\mathrm{Tf}$ ) were potentially useful as surrogates for improved glycemia, six biomarkers (including serum RPB4 and modified apo-AI) increased expression as a consequence of anakinra treatment and four proteins were candidate biomarkers for a glycemic response to anakinra treatment. Furthermore, we found increased RBP4 to be associated with improved beta cell secretory function and increased TTR, RBP4 and modified apo-AI to be associated with decreased inflammation. Apo-AI and Tf are important anti-atherogenic factors. Taken together, our results suggest that the anakinrainduced changes in the serum proteome pool are associated with a decreased CVD risk, reduced inflammation and improved beta cell secretory function. These hypotheses need to be tested in prospective studies.

Acknowledgements This work was supported by funding from the Danish Diabetes Association, The Danish Council for Independent Research, Medical Sciences, Steno Diabetes Center and Novo Nordisk A/S. We thank Zohreh Sattari, Hanne Foght and Ellis D. Schjerning for their skilful technical assistance in the laboratory.

\section{References}

1. Mandrup-Poulsen T. The role of interleukin-1 in the pathogenesis of IDDM. Diabetologia. 1996;39:1005-29.

2. Larsen CM, Faulenbach M, Vaag A, et al. Interleukin-1-receptor antagonist in type 2 diabetes mellitus. $\mathrm{N}$ Engl J Med. 2007;356:1517.

3. Maedler K, Sergeev P, Ris F, et al. Glucose-induced B cell production of IL-1ß contributes to glucotoxicity in human pancreatic islets. J Clin Investig. 2002;110:851-60.

4. Zhou R, Tardivel A, Thorens B, Choi I, Tschopp J. Thioredoxininteracting protein links oxidative stress to inflammasome activation. Nat Immunol. 2009;11:136-40.

5. Maedler K, Sergeev P, Ehses JA, et al. Leptin modulates $\beta$ cell expression of IL-1 receptor antagonist and release of IL-1ß in human islets. Proc Natl Acad Sci. 2004;101:8138-43.

6. Berchtold LA, Larsen CM, Vaag A, et al. IL-1 receptor antagonism and muscle gene expression in patients with type 2 diabetes. Eur Cytokine Netw. 2009;20:81-7.

7. Larsen CM, Faulenbach M, Vaag A, Ehses JA, Donath MY, Mandrup-Poulsen T. Sustained effects of interleukin-1 receptor antagonist treatment in type 2 diabetes. Diab Care. 2009;32:1663.

8. Qu Y, Adam BL, Yasui Y, et al. Boosted decision tree analysis of surface-enhanced laser desorption/ionization mass spectral serum profiles discriminates prostate cancer from noncancer patients. In: Am Assoc Clin Chem; 2002. p. 1835-43.

9. Simonsen AH, McGuire J, Hansson O, et al. Novel panel of cerebrospinal fluid biomarkers for the prediction of progression to Alzheimer dementia in patients with mild cognitive impairment. Arch Neurol. 2007;64:366-70.

10. Sundsten T, Eberhardson M, Goransson M, Bergsten P. The use of proteomics in identifying differentially expressed serum proteins in humans with type 2 diabetes. Proteome Sci. 2006;4:22.

11. Sundsten T, Zethelius B, Berne C, Bergsten P. Plasma proteome changes in subjects with type 2 diabetes mellitus with a low or high early insulin response. Clin Sci. 2008;114:499-507.

12. Allard L, Lescuyer P, Burgess J, et al. ApoC-I and ApoC-III as potential plasmatic markers to distinguish between ischemic and hemorrhagic stroke. Proteomics. 2004;4:2242-51.

13. Chen Y-D, Zheng S, Yu J-K, Hu X. Artificial neural networks analysis of surface-enhanced laser desorption/ionization mass spectra of serum protein pattern distinguishes colorectal cancer from healthy population. Clin Cancer Res. 2004;10:8380-5. 
14. Li J, Zhang Z, Rosenzweig J, Wang YY, Chan DW. Proteomics and bioinformatics approaches for identification of serum biomarkers to detect breast cancer. Clin Chem. 2002;48:1296-304.

15. Nomura F, Tomonaga T, Sogawa K, et al. Identification of novel and downregulated biomarkers for alcoholism by surface-enhanced laser desorption/ionization-mass spectrometry. Proteomics. 2004;4:1187-94.

16. Petricoin EF, Ardekani AM, Hitt BA, et al. Use of proteomic patterns in serum to identify ovarian cancer. Lancet. 2002;359:572-7.

17. Petricoin III EF, Ornstein DK, Paweletz CP, et al. Serum proteomic patterns for detection of prostate cancer. J Natl Cancer Inst. 2002;94:1576-8.

18. Uchida T, Fukawa A, Uchida M, Fujita K, Saito K. Application of a novel protein biochip technology for detection and identification of rheumatoid arthritis biomarkers in synovial fluid. J Proteome Res. 2002;1:495-9.

19. Koenig RJ, Peterson CM, Jones RL, Saudek C, Lehrman M, Cerami A. Correlation of glucose regulation and hemoglobin AIc in diabetes mellitus. N Engl J Med. 1976;295:417.

20. Timms JF, Arslan-Low E, Gentry-Maharaj A, et al. Preanalytic influence of sample handling on SELDI-TOF serum protein profiles. Clin Chem. 2007;53:645-56.

21. Albrethsen J, Bøgebo R, Olsen J, Raskov H, Gammeltoft S. Preanalytical and analytical variation of surface-enhanced laser desorption-ionization time-of-flight mass spectrometry of human serum. Clin Chem Lab Med. 2006;44:1243-52.

22. Basu TK, Basualdo C. Vitamin A homeostasis and diabetes mellitus. Nutrition. 1997;13:804-6.

23. Refai E, Dekki N, Yang SN, et al. Transthyretin constitutes a functional component in pancreatic $\beta$-cell stimulus-secretion coupling. Proc Natl Acad Sci. 2005;102:17020-5.

24. Sundsten T, Ostenson CG, Bergsten P. Serum protein patterns in newly diagnosed type 2 diabetes mellitus-influence of diabetic environment and family history of diabetes. Diabetes/Metab Res Rev. 2008;24(2):148-54.

25. Myron Johnson A, Merlini G, Sheldon J, Ichihara K. Clinical indications for plasma protein assays: transthyretin (prealbumin) in inflammation and malnutrition. Clin Chem Lab Med: CCLM/ FESCC. 2007;45:419.

26. Perez Valdivieso JR, Bes-Rastrollo M, Monedero P, de Irala J, Lavilla FJ. Impact of prealbumin levels on mortality in patients with acute kidney injury: an observational cohort study. J Renal Nutr. 2008;18:262-8.

27. Raghu P, Sivakumar B. Interactions amongst plasma retinolbinding protein, transthyretin and their ligands: implications in vitamin A homeostasis and transthyretin amyloidosis. BBAProteins and Proteomics. 2004;1703:1-9.

28. Lu J, Dixon WT, Tsin AT, Basu TK. The metabolic availability of vitamin $\mathrm{A}$ is decreased at the onset of diabetes in BB rats. J Nutr. 2000;130:1958-62.

29. Tuitoek PJ, Rittere SJ, Smitha JE, Basu TK. Streptozotocininduced diabetes lowers retinol-binding protein and transthyretin concentrations in rats. Br J Nutr. 2007;76:891-7.

30. Kemp SF, Frindik JP. Effect of metabolic control on serum protein concentrations in diabetes. Acta Paediatr. 1991;80:938-43.

31. Janke J, Engeli S, Boschmann M, et al. Retinol-binding protein 4 in human obesity. Diabetes. 2006;55:2805-10.

32. Kanaka-Gantenbein C, Margeli A, Pervanidou P, et al. Retinolbinding protein 4 and lipocalin- 2 in childhood and adolescent obesity: when children are not just "Small Adults". Clin Chem. 2008;54:1176.

33. Krzyzanowska K, Zemany L, Krugluger W, et al. Serum concentrations of retinol-binding protein 4 in women with and without gestational diabetes. Diabetologia. 2008;51:1115-22.

34. Graham TE, Yang Q, Bluher M, et al. Retinol-binding protein 4 and insulin resistance in lean, obese, and diabetic subjects. N Engl J Med. 2006;354:2552.

35. Lee DC, Lee JW, Im JA. Association of serum retinol binding protein 4 and insulin resistance in apparently healthy adolescents. Metabolism. 2007;56:327-31.

36. Balagopal P, Graham TE, Kahn BB, Altomare A, Funanage V, George D. Reduction of elevated serum retinol binding protein in obese children by lifestyle intervention: association with subclinical inflammation. J Clin Endocrinol Metab. 2007;92:1971.

37. Choi SH, Kwak SH, Youn BS, et al. High plasma retinol binding protein-4 and low plasma adiponectin concentrations are associated with severity of glucose intolerance in women with previous gestational diabetes mellitus. J Clin Endocrinol Metab. 2008;93:3142.

38. Ribel-Madsen R, Friedrichsen M, Vaag A, Poulsen P. Retinolbinding protein 4 in twins. Diabetes. 2009;58:54-60.

39. Oram JF, Yokoyama S. Apolipoprotein-mediated removal of cellular cholesterol and phospholipids. J Lipid Res. 1996;37:2473-91. 